\title{
Introduction
}

\section{Inflammatory Breast Cancer}

\author{
Sandra M. Swain \\ Breast Cancer Section, Medical Oncology Branch, Center for Cancer Research, National Cancer Institute, \\ Bethesda, MD 20889, USA
}

Inflammatory breast cancer has been recognized as a distinct entity for decades, but the precise definition still remains elusive. It is most likely a heterogenous group of diseases, as is true with breast cancer overall. However, one aspect is clear, patients with this diagnosis have an aggressive form of breast cancer and a poorer overall survival. If we look at the characteristics of the tumors found when a diagnosis of inflammatory breast cancer is made, they are not at all consistent. Some tumors have hormone receptor positivity, others lack HER2 gene amplification, and some patients have dermal lymphatic invasion. One characteristic that is present in most patients is lymph node involvement and a higher proportion with metastatic disease at diagnosis. This issue of Breast Disease is a comprehensive review of all aspects of inflammatory breast cancer from the epidemiology, biology, clinical diagnosis, local, and systemic treatment. These papers also pose the interesting and important future questions for the study of inflammatory breast cancer. 